\title{
Promoción de estrategias de aprendizaje desde el accionar docente: percepciones a nivel universitario
}

Promotion of learning strategies from teaching action: university perceptions of teaching

\section{Volumen 18, Número 3 \\ Setiembre-Diciembre}

pp. 1-20

Este número se publica el 1 de setiembre de 2018

DOI: https://doi.org/10.15517/aie.v18i3.34099

\author{
Patricio Henríquez Ritchie \\ Miriam Álvarez Mariscal
}

Revista indizada en REDALYC, $\underline{\text { SCIELO }}$

Revista distribuida en las bases de datos:

LATINDEX, DOAJ, REDIB, IRESIE, CLASE, DIALNET, SHERPA/ROMEO, QUALIS-CAPES, MIAR

Revista registrada en los directorios:

ULRICH'S $, \underline{R E D I E}, \underline{R I N A C E}, \underline{\text { OEI }}, \underline{\text { MAESTROTECA, PREAL, }}$ 


\title{
Promoción de estrategias de aprendizaje desde el accionar docente: percepciones a nivel universitario \\ Promotion of learning strategies from teaching action: university perceptions of teaching
}

\author{
Patricio Henríquez Ritchie ${ }^{1}$ \\ Miriam Álvarez Mariscal
}

\begin{abstract}
Resumen: En este artículo se exponen los resultados de una investigación cuyo objetivo general fue explorar las estrategias educativas utilizadas por docentes universitarios, las formas de evaluación del aprendizaje y las estrategias de aprendizaje que promueven en sus estudiantes. Se eligieron dos contextos relacionados con la formación de agentes educativos: la licenciatura en Ciencias de la Educación de la Universidad Autónoma de Baja California (UABC) y la Facultad de Educación de la Universidad Complutense de Madrid (UCM). Se seleccionó una muestra intencional no representativa de 24 docentes (19 docentes de UCM y 5 docentes de $(U A B C)$, a quienes se les aplicó una entrevista en profundidad semi-estructurada. En el presente artículo se exponen los resultados relacionados con la dimensión de estrategias de aprendizaje; al respecto, se identificó una subdimensión relacionada con la promoción/fomento de estrategias de aprendizaje, tres categorías (aprendizaje centrado en el estudiante, aprendizaje centrado en los contenidos y aprendizaje centrado en el docente) y cuatro sub-categorías de análisis. A partir de los resultados de la investigación, se sugiere profundizar la comprensión de las estrategias de aprendizaje centradas en los estudiantes, las cuales se identifican con las teorías constructivistas de generación del conocimiento. A su vez, se espera que estos resultados aporten elementos teóricos y metodológicos para mejorar la comprensión en torno a las estrategias de aprendizaje que utilizan actualmente los estudiantes universitarios en contextos de formación de agentes educativos y sus procesos metacognitivos.
\end{abstract}

Palabras clave: método de enseñanza, estrategias educativas, método de aprendizaje, enseñanza superior

Abstract: In this article are exposed the results of a research whose objective was to explore the educational strategies used by university teachers, the forms of evaluation of learning and the strategies of learning that promote in their students. Two contexts related to the training of educational agents were chosen: the degree of Science in Education of the Autonomous University of Baja California (UABC) and the Faculty of Education of the Universidad Complutense de Madrid (UCM). A non-representative sample of 24 teachers (19 UCM teachers and 5 $U A B C$ teachers) was selected, who were interviewed in a semi-structured depth. This article presents the results related to the dimension of learning strategies: in this respect, a sub-dimension related to the promotion of learning strategies was identified, three categories (student-centered learning, content-centered learning and teachercentered learning) and four sub-categories of analysis. From the results of the research, it is suggested to deepen the understanding of student-centered learning strategies, which are identified with the constructivist theories of knowledge generation. At the same time, these results are expected to provide theoretical and methodological elements to improve the understanding of the learning strategies currently used by university students in contexts of formation of educational agents and their metacognitive processes.

Key words: teaching methods, educational strategies, learning methods, higher education

\footnotetext{
1 Docente e Investigador de la Universidad Autónoma de Baja California, México.Dirección elecrónica: phennriquez@uabc.edu.mx

2 Docente e Investigadora de la Universidad Autónoma de Baja California, México.Dirección elecrónica: miriam3@uabc.edu.mx
}

Artículo recibido: 30 de noviembre, 2017

Enviado a corrección: 16 de abril, 2018

Aprobado: 25 de junio, 2018 


\section{Introducción}

¿Qué estrategias de aprendizaje promueven actualmente los docentes universitarios en contextos de formación de futuros agentes educativos? ¿Cuáles son los fundamentos teóricos que sustentan la acción de enseñanza del docente y el fomento de estrategias de aprendizaje en sus estudiantes? ¿Existe una alineación entre las estrategias de enseñanza que utilizan los docentes y las estrategias de aprendizaje que intentan promover en sus estudiantes? Estas son algunas de las preguntas que guían el interés de la investigación reportada en el presente documento.

Respecto a las interrogantes anteriores, existe una multiplicidad insoslayable de definiciones y perspectivas desde las cuales se han analizado las estrategias de aprendizaje, su uso, aplicación y fomento desde la acción docente (Donche, De Maeyer, Coertjens, Val Daal y Van Petegem, 2013; Fernández, Pérez, Peña y Mercado, 2011; García y Ortega, 2012; García, Sánchez, Jiménez y Gutiérrez, 2012; Gastón, Martínez y Martín, 2016; Kamboj y Kumar, 2015; León, Risco y Alarcón, 2014; Rodríguez, Sanmiguel y Hernández, 2012; Shukla y Dungsungnoen, 2016). Estos estudios se han llevado a cabo en diferentes contextos y niveles educativos, centran su interés principalmente en el análisis de las estrategias de aprendizaje utilizadas por los estudiantes, la influencia de las estrategias de enseñanza de los docentes y su impacto en variables de rendimiento académico.

Junto a la diversidad de perspectivas de análisis en torno a las estrategias de aprendizaje, el presente documento intenta analizar la identificación de dichas estrategias con ciertas posturas epistemológicas acerca de la generación del conocimiento en contextos educativos, específicamente, teorías psicológicas de aprendizaje. En ese sentido, tal como lo destaca Hernández Rojas (1998), a partir de los principales lineamientos teóricos y las implicaciones pedagógicas de estas teorías se puede identificar la asignación de roles específicos en docentes y estudiantes que derivan en el uso de determinadas estrategias de enseñanza y aprendizaje, entendidas estas últimas como un binomio inseparable tanto en lo conceptual como en lo procedimental.

El objetivo principal de este estudio es explorar comprensivamente la percepción de los docentes universitarios con respecto a las estrategias de aprendizaje que intentan promover/fomentar en sus estudiantes, a partir de su acción de enseñanza. Para lograr este propósito, se entrevistó a los docentes de tiempo completo de la licenciatura en Ciencias de la Educación de la Universidad Autónoma de Baja California (UABC) y de la Facultad de Educación de la Universidad Complutense de Madrid (UCM), quienes tienen a cargo la 
formación universitaria de futuros agentes educativos (docentes, directivos, investigadores, administradores, entre otros).

\section{Referentes teóricos}

Las estrategias de aprendizaje han sido definidas y analizadas desde múltiples perspectivas. Algunos autores, como Díaz y Hernández (2002), las comprenden como aquellos procedimientos conscientes, controlados y voluntarios que ejecutan los estudiantes para lograr ciertos objetivos, previamente establecidos por los docentes. De esta manera, se comprenden de la mano con la planificación de enseñanza de los docentes, quienes podrían promover, por ejemplo, la repetición o retención de los contenidos vistos en clases, el logro de aprendizajes significativos, la capacidad de resolución de situaciones problemáticas o la comprensión de los contenidos con base en el trabajo grupal colaborativo entre los estudiantes.

Otros autores (Rodríguez et al., 2012; García y Ortega, 2012; García et al. 2012) prefieren hablar de "estilos de aprendizaje" para referirse a las maneras en que los estudiantes incorporan los contenidos educativos según sus capacidades y características cognitivas. Específicamente, los estilos de aprendizaje remiten a la manera en que los individuos concentran, procesan, internalizan y recuerdan la información (contenidos) enseñada por sus docentes (Kamboj y Kumar, 2015). En este sentido, algunos identifican estilos de aprendizaje activos, reflexivos, teóricos y pragmáticos según el rol que jueguen los estudiantes en su aprendizaje y la manera en que abordan los contenidos educativos (Rodríguez et al., 2012). Otros, por su parte, se centran en la manera en que cada estudiante procesa la información enseñada por sus docentes, de acuerdo con estilos de aprendizaje visual, auditivo o kinestésico (García y Ortega, 2012).

Asimismo, Kablan y Kaya (2014) destacan la persistencia actual de prácticas pedagógicas tradicionales basadas en la transmisión de conocimientos del docente a los estudiantes y abogan por la promoción de prácticas constructivistas en las que los mismos estudiantes protagonizan los procesos de construcción de conocimientos. En este sentido, proponen la consideración de estilos de aprendizaje según la manera en que los sujetos crean significados en torno a los contenidos educativos: experiencia concreta, observación reflexiva, conceptualización abstracta y experimentación activa.

En congruencia con lo anterior, respecto a los paradigmas psicológicos en educación, Hernández Rojas (1998) destaca los principales lineamientos teóricos y las implicaciones 
pedagógicas de cada uno describiendo los roles que deben jugar docentes y estudiantes según cada perspectiva. Tal como lo destaca el autor, estas vertientes teóricas difieren en algunos aspectos referidos al proceso de comprensión de la realidad en los individuos y las formas de construcción de conocimientos en el marco de las prácticas educativas. En la Tabla 1 se pueden observar los principales postulados de cada paradigma:

Tabla 1.

Paradigmas psicológicos en educación. Definiciones en torno a la enseñanza y el aprendizaje

\begin{tabular}{|c|c|c|c|c|}
\hline Conductismo & Humanismo & Cognitivismo & $\begin{array}{c}\text { Teoría } \\
\text { psicogenética }\end{array}$ & Teoría sociocultural \\
\hline \multicolumn{5}{|c|}{ ENSEÑANZA Y ROL DEL DOCENTE } \\
\hline $\begin{array}{l}\text { - Enseñanza: } \\
\text { conjunto } \\
\text { adecuado de } \\
\text { contingencias de } \\
\text { reforzamiento y } \\
\text { estímulos } \\
\text { - Proceso de } \\
\text { transmisión de } \\
\text { contenidos: } \\
\text { docente- } \\
\text { estudiantes } \\
\text { - Objetivos } \\
\text { relacionados con } \\
\text { conductas } \\
\text { observables de } \\
\text { estudiantes } \\
\text { - Estrategias: } \\
\text { enseñanza } \\
\text { programada } \\
\text { - Evaluación: } \\
\text { pruebas } \\
\text { objetivas }\end{array}$ & $\begin{array}{l}\text { - Enseñanza: } \\
\text { medio de } \\
\text { autorrealización, } \\
\text { estudiantes con } \\
\text { iniciativa y } \\
\text { espíritu } \\
\text { colaborativo } \\
\text { - Enseñanza } \\
\text { indirecta: } \\
\text { contexto de } \\
\text { libertad para el } \\
\text { desarrollo pleno } \\
\text { (cognitivo y } \\
\text { afectivo) } \\
\text { - Estrategias: } \\
\text { flexibilidad, } \\
\text { centradas en el } \\
\text { estudiante } \\
\text { - Evaluación: } \\
\text { autoevaluación }\end{array}$ & $\begin{array}{l}\text { - Enseñanza: } \\
\text { proceso de } \\
\text { entrega de } \\
\text { contenidos } \\
\text { valorados } \\
\text { culturalmente } \\
\text { - Proceso de } \\
\text { planificación y } \\
\text { organización de } \\
\text { los contenidos } \\
\text { según } \\
\text { características } \\
\text { cognitivas y } \\
\text { motivacionales } \\
\text { de estudiantes } \\
\text { - Estrategias: } \\
\text { aprender a } \\
\text { aprender y } \\
\text { enseñar a } \\
\text { pensar } \\
\text { - Evaluación: } \\
\text { cualitativa }\end{array}$ & $\begin{array}{l}\text { - Enseñanza: apoyo } \\
\text { sistemático, } \\
\text { sostenido y ajustado } \\
\text { según niveles } \\
\text { cognitivos de los } \\
\text { estudiantes } \\
\text { - Contexto: } \\
\text { situaciones y } \\
\text { experiencias } \\
\text { relevantes } \\
\text { - Objetivos: potenciar } \\
\text { el desarrollo general } \\
\text { de los estudiantes } \\
\text { - Estrategias: facilitar } \\
\text { la interpretación y } \\
\text { reconstrucción de los } \\
\text { contenidos } \\
\text { - Evaluación } \\
\text { centrada en los } \\
\text { procesos (formativa) }\end{array}$ & $\begin{array}{l}\text { - Enseñanza: proceso } \\
\text { de transmisión de } \\
\text { instrumentos } \\
\text { socioculturales } \\
\text { (conocimientos, } \\
\text { valores, actitudes, } \\
\text { normas) } \\
\text { - Docentes como } \\
\text { agentes educativos y } \\
\text { mediadores culturales } \\
\text { - Objetivos en función } \\
\text { de los instrumentos } \\
\text { con valor cultural } \\
\text { - Estrategias: } \\
\text { promoción de la } \\
\text { interacción social, } \\
\text { participación e } \\
\text { involucramiento } \\
\text { - Evaluación dinámica } \\
\text { (procesos) e } \\
\text { interactiva }\end{array}$ \\
\hline \multicolumn{5}{|c|}{ APRENDIZAJE Y ROL DEL ESTUDIANTE } \\
\hline $\begin{array}{l}\text { - Aprendizaje: } \\
\text { cambios en la } \\
\text { conducta de los } \\
\text { estudiantes } \\
\text { - Proceso } \\
\text { moldeado desde } \\
\text { el exterior: } \\
\text { métodos, } \\
\text { contenidos, } \\
\text { situaciones } \\
\text { instruccionales } \\
\text { - Estudiante: } \\
\text { actor pasivo del } \\
\text { proceso } \\
\text { educativo }\end{array}$ & $\begin{array}{l}\text { - Aprendizaje: } \\
\text { proceso innato } \\
\text { de desarrollo } \\
\text { integral } \\
\text { - Proceso } \\
\text { experiencial y } \\
\text { participativo: } \\
\text { relacionado con } \\
\text { aspectos } \\
\text { personales y } \\
\text { centrado en } \\
\text { estudiantes }\end{array}$ & $\begin{array}{l}\text { - Aprendizaje } \\
\text { significativo: } \\
\text { vinculación de } \\
\text { conocimientos } \\
\text { previos y } \\
\text { nuevos } \\
\text { - Pluralidad de } \\
\text { aprendizajes: } \\
\text { distintos ritmos } \\
\text { de aprendizaje }\end{array}$ & $\begin{array}{l}\text { - Aprendizaje: } \\
\text { proceso de } \\
\text { significación de los } \\
\text { contenidos } \\
\text { educativos } \\
\text { - Aprendizaje en } \\
\text { función de desarrollo } \\
\text { cognitivo } \\
\text { - Estudiante como } \\
\text { constructor activo de } \\
\text { su propio } \\
\text { conocimiento y } \\
\text { reconstructor de los } \\
\text { contenidos escolares }\end{array}$ & $\begin{array}{l}\text { - Aprendizaje como } \\
\text { proceso social de } \\
\text { adquisición de } \\
\text { instrumentos } \\
\text { socioculturales } \\
\text { - Aprendizaje como } \\
\text { proceso } \\
\text { inherentemente } \\
\text { interactivo y dialógico } \\
\text { - Estudiante como ser } \\
\text { social, producto de } \\
\text { las interacciones } \\
\text { sociales con sus } \\
\text { pares y adultos }\end{array}$ \\
\hline
\end{tabular}

Fuente: Hernández Rojas (1998) 
De esta manera, se puede observar que cada paradigma propone distintas formas de comprender los procesos educativos y, por ende, de llevar a cabo las actividades escolares. De esto derivan distintas maneras de comprender las estrategias de enseñanza-aprendizaje y distintas miradas en torno a los roles asignados a docentes y estudiantes. Asimismo, cada paradigma traza sus propios objetivos para las prácticas de enseñanza y define los procesos evaluativos en función de dichos objetivos.

En el presente artículo se retoman las consideraciones teóricas aportadas por Coll (2004) respecto a los elementos principales que componen el acto educativo, entendido a partir de las prácticas educativas formales. En efecto, según su concepción constructivista de la enseñanza y el aprendizaje, la educación se debe comprender como una práctica social con una función inherentemente socializadora en los individuos, en función de su desarrollo personal a través de procesos de individualización (construcción de la identidad personal) y socialización (incorporación a un contexto social y cultural). Con base en lo anterior, describe la especificidad de las situaciones de enseñanza-aprendizaje a partir del "triángulo didáctico o interactivo" compuesto por tres vértices: i) los contenidos educativos (objeto de la enseñanza-aprendizaje), ii) proceso de aprendizaje de los estudiantes (construcción de significados y atribución de sentido en torno a los contenidos) y iii) proceso de enseñanza de los docentes (ayuda sistemática, sostenida y ajustada al aprendizaje). En el presente documento se comprenden las estrategias de aprendizaje según el punto neurálgico en el que los docentes basan su promoción: i) estrategias basadas en la actividad de aprendizaje de los estudiantes, ii) en la naturaleza de los contenidos enseñados o iii) en la actividad de enseñanza de los profesores.

En síntesis, se arguye la imposibilidad de comprender de manera independiente y autónoma las estrategias de aprendizaje de los estudiantes respecto a la acción de enseñanza del docente. Así, en concordancia con algunos autores (Donche, De Maeyer y Van Petegem, 2007; Monereo, Castelló, Clariana, Palma y Pérez, 2007; Shukla y Dungsungnoen, 2016), no se puede soslayar el hecho de que las estrategias de enseñanza impactan profundamente el aprendizaje de los estudiantes, de manera que los hábitos y técnicas de estudio que componen la acción estratégica del educando están influidas por la acción estratégica del agente de enseñanza. En otras palabras, los estudiantes utilizan las estrategias de aprendizaje que mejor se acomoden a las estrategias de enseñanza y de evaluación que utilizan sus profesores. En este artículo se exploran las percepciones de los docentes universitarios en torno a la promoción de estrategias de aprendizaje en sus 
estudiantes. Para esto, se recurrió a los docentes que tienen a cargo la formación de los futuros agentes educativos, los cuales intervendrán directamente en los procesos educativos de las próximas generaciones.

\section{Método}

La investigación expuesta en el presente documento fue de tipo cualitativa. En este sentido, se adhiere al paradigma interpretativo que apunta a comprender las realidades sociales desde las percepciones de los sujetos que las conforman, soslayan la perspectiva de la realidad como un objeto observable, medible y ajeno al observador: en el campo de la educación, apunta a la comprensión de los fenómenos educativos de manera intersubjetiva, holística e interactiva (Carbajosa, 2011). En el presente estudio, se optó por un acercamiento a las subjetividades de los participantes a través de las entrevistas semiestructuradas en profundidad.

\subsection{Contexto espacio-temporal}

La investigación consideró dos instituciones de educación superior que ofertan programas de formación de agentes educativos: i) la Universidad Autónoma de Baja California (UABC), Facultad de Ciencias Administrativas y Sociales (FACYS), licenciatura en Ciencias de la Educación, y ii) la Universidad Complutense de Madrid (UCM), Facultad de Educación. Por un lado, la licenciatura en Ciencias de la Educación de la UABC promueve la formación de agentes educativos enfocados en áreas, tales como: la docencia, investigación, gestión y administración de instituciones educativas. Por otro lado, la Facultad de Educación de la UCM está conformada por 9 departamentos de didáctica (lengua y literatura; ciencias experimentales; ciencias sociales; matemáticas; didáctica y organización escolar; expresión musical y corporal; métodos de investigación y diagnóstico en educación; psicología evolutiva y de la educación; y teoría e historia de la educación), los cuales apuntan específicamente a la formación de futuros docentes.

A su vez, el proyecto se llevó a cabo durante el período 2016-17.

\subsection{Participantes}

La licenciatura en Ciencias de la Educación de la UABC consta de 6 docentesinvestigadores de tiempo completo, los cuales ejecutan labores de docencia, investigación y coordinación de programas académicos. Se cuenta con 19 docentes de asignatura que 
cumplen funciones exclusivamente de docencia. En este estudio se seleccionó una muestra intencional no probabilística de 4 docentes de tiempo completo y 1 docente de asignatura de este programa educativo de la UABC.

Por su parte, los departamentos de didáctica de la Facultad de Educación de la UCM están conformados por un promedio de 25 docentes-investigadores de tiempo completo cada uno (es decir, un total aproximado de 225 docentes en toda la Facultad), de los cuales se seleccionó una muestra intencional no probabilística de 19 docentes. En síntesis, la muestra intencional no probabilística incluyó un total de 24 docentes entre ambas instituciones universitarias (ver Tabla 2):

Tabla 2.

Departamentos y participantes de la Facultad de Educación (UCM) y de la licenciatura en Ciencias de la Educación (UABC)

\begin{tabular}{|c|c|}
\hline DEPARTAMENTOS (UCM) & PARTICIPANTES \\
\hline Didáctica de las Ciencias Sociales (CCSS) & 3 \\
\hline Didáctica de las Ciencias Experimentales (CCEE) & 2 \\
\hline Didáctica de la Lengua y Literatura (LENGUA Y LIT) & 3 \\
\hline Didáctica de las Matemáticas (MAT) & 2 \\
\hline Didáctica y Organización Escolar (DIDAC) & 2 \\
\hline Teoría e Historia de la Educación (TEO E HISTO) & 3 \\
\hline Expresión Musical y Corporal (MUS Y CORP) & 1 \\
\hline Métodos de Investigación y Diagnóstico en Educación (MIDE) & 3 \\
\hline Psicología Evolutiva y de la Educación (PSICO Y EVO) & 1 \\
\hline SUBTOTAL & 19 \\
\hline Lic. en Ciencias de la Educación (UABC) & 5 \\
\hline TOTAL & 24 \\
\hline
\end{tabular}

Fuente: Elaboración propia (2017)

Cabe destacar que, en el marco de la selección intencional de la muestra de estudio, se informó a los participantes acerca de la confidencialidad de la información que facilitaron, de manera que los reportes de investigación generados a partir del análisis de los resultados no individualizarán a los docentes involucrados.

\subsection{Instrumentos}

La entrevista en profundidad fue la técnica de recolección de datos utilizada en esta investigación, ya que se comprende como una herramienta idónea para la exploración de dimensiones subjetivas (percepciones, valoraciones, proyecciones, creencias) de los sujetos participantes en estudios sociales. Paralelamente, según algunos autores (Robles, 2011; Vargas Jiménez, 2012) convierte a los entrevistados en interlocutores sociales a través de la 
acción comunicativa con el investigador, lo cual permite la interpretación de la realidad social investigada.

De esta manera, se elaboró una pauta de entrevista semiestructurada para guiar el diálogo con los entrevistados sobre la base de los objetivos del estudio, la cual constó de 3 dimensiones y 6 subdimensiones (ver Tabla 3):

Tabla 3.

Pauta de entrevista semiestructurada para docentes

\begin{tabular}{|c|c|c|}
\hline Dimensión & Subdimensión & Preguntas \\
\hline \multirow[t]{2}{*}{$\begin{array}{l}\text { Estrategias } \\
\text { de } \\
\text { enseñanza }\end{array}$} & $\begin{array}{l}\text { Definición y } \\
\text { uso }\end{array}$ & $\begin{array}{l}\text { - ¿Cómo define el concepto "estrategias de enseñanza"? } \\
\text { - ¿Cuáles son las principales estrategias de enseñanza que } \\
\text { - ¿Qtiliza como docente para impartir su materia? } \\
\text { ¿enseñanza? } \\
\text { - ¿Qué herramientas/instrumentos/recursos utiliza como } \\
\text { apoyo? } \\
\text { - ¿De qué manera influye el tamaño del grupo (cantidad) de } \\
\text { estudiantes en las estrategias de enseñanza que utiliza? }\end{array}$ \\
\hline & $\begin{array}{c}\text { Factores } \\
\text { institucionales }\end{array}$ & $\begin{array}{l}\text { - ¿Cuáles son las instancias de capacitación/promoción en } \\
\text { torno a estrategias de enseñanza desde la universidad en } \\
\text { la planta docente de la licenciatura/facultad? } \\
\text { ¿Cuál es la proporción de los docentes de tiempo respecto } \\
\text { a los docentes de "asignatura" que trabajan en la } \\
\text { licenciatura/facultad? ¿Cuál es su opinión al respecto? }\end{array}$ \\
\hline $\begin{array}{l}\text { Estrategias } \\
\text { de } \\
\text { aprendizaje }\end{array}$ & $\begin{array}{l}\text { Promoción y } \\
\text { fomento }\end{array}$ & $\begin{array}{l}\text { - ¿Qué estrategias de aprendizaje intenta promover en sus } \\
\text { estudiantes? } \\
\text { ¿Cómo se integran las estrategias de enseñanza que utiliza } \\
\text { con las estrategias de aprendizaje que promueve en sus } \\
\text { estudiantes? }\end{array}$ \\
\hline \multirow{3}{*}{$\begin{array}{l}\text { Evaluación } \\
\text { del } \\
\text { aprendizaje }\end{array}$} & Funciones & $\begin{array}{l}\text { Según su opinión, ¿Cuáles son las funciones que tiene } \\
\text { (debe tener) la evaluación dentro del proceso de enseñanza- } \\
\text { aprendizaje? }\end{array}$ \\
\hline & Estrategias & $\begin{array}{l}\text { - ¿Cuáles son las estrategias de evaluación que utiliza en el } \\
\text { marco de su práctica de enseñanza? ¿Con qué finalidad? } \\
\text { - ¿De qué manera afecta tamaño del grupo (cantidad) de } \\
\text { estudiantes en las estrategias de evaluación que utiliza? }\end{array}$ \\
\hline & Instrumentos & $\begin{array}{l}\text { - ¿Qué instrumentos de evaluación utiliza en su práctica } \\
\text { docente? }\end{array}$ \\
\hline
\end{tabular}

Fuente: Elaboración propia (2017)

\subsection{Procedimiento}

Las fases seguidas en el desarrollo de la investigación fueron las siguientes:

- Desarrollo de antecedentes y marco teórico (septiembre-octubre 2016). Elaboración del marco teórico del estudio, estructuración de las bases conceptuales y definición de los conceptos claves. 
- Aplicación de entrevistas en profundidad (noviembre 2016-febrero 2017). En una primera instancia, se aplicaron las entrevistas en la licenciatura en Ciencias de la Educación de la FCAYS de la UABC. Posteriormente, durante enero-febrero de 2017, se realizaron las entrevistas a los docentes de Facultad de Educación de la UCM. A nivel general, las entrevistas tuvieron un tiempo de duración de 40 minutos aproximadamente cada una.

- Análisis de resultados (marzo-agosto 2017). Durante esta última etapa se realizaron los análisis cualitativos de los datos recolectados a través de las entrevistas.

\subsection{Análisis de datos}

Se utilizó la técnica de análisis de contenido. Esta herramienta metodológica permite interpretar los datos identificando códigos y categorías de análisis en los discursos de los participantes. Según Piñuel Raigada (2002), se trata de un conjunto de procedimientos interpretativos de productos comunicativos (mensajes, textos, discursos) originados en interacciones dialógicas registradas previamente. Las etapas seguidas en el análisis cualitativo de la información fueron:

- Captura de la información mediante grabaciones de audio de las entrevistas.

- Realización de transcripciones.

- Análisis de las opiniones, percepciones y discursos de los entrevistados.

- Identificación de categorías (clasificación conceptual de unidades temáticas cubiertas bajo un mismo tópico) y códigos (conjunto de unidades de texto que corresponden dentro de una categoría) de análisis.

\section{Resultados}

A partir del análisis de la información recolectada se identificó una subdimensión relacionada con las estrategias de aprendizaje que los docentes intentar fomentar en sus estudiantes a partir de su acción de enseñanza. Dentro esta subdimensión, se identificaron 3 categorías de análisis dependiendo del centro de interés en el que el docente se sitúa para promover el uso de estrategias de aprendizaje: i) aprendizaje centrado en el estudiante, ii) aprendizaje centrado en los contenidos y iii) aprendizaje centrado en el docente.

4.1 Aprendizaje centrado en el estudiante: según la percepción de algunos docentes entrevistados, se podría establecer una asociación entre el uso de ciertas estrategias de 
aprendizaje y el perfil cognitivo del estudiantado, independientemente de los contenidos enseñados. En efecto, algunos docentes permiten un grado de libertad para que los estudiantes elijan las estrategias más idóneas a sus características cognitivas personales, acercándose a lo que algunos autores han estudiado bajo el concepto de "metacognición". Precisamente, este concepto surge a partir de la perspectiva constructivista de que la acción de enseñanza de los docentes debe enfocarse en lograr que los estudiantes sean capaces de "aprender a aprender", lo cual implica que sean capaces de identificar las estrategias de aprendizaje y técnicas de estudio afines a sus propios perfiles cognitivos.

De esta manera, desde la mirada de algunos docentes se vislumbra una postura flexible y adaptativa a la multiplicidad de estilos cognitivos de sus estudiantes:

[...] Cada uno según sus gustos y habilidades creará su estilo, cogerá su oficio y lo hará tan bien como pueda... Tengo alumnos que el trabajo de grupo les pone enfermos, en cambio tengo otros que si no es en grupo se desorientan, así es que cada uno hace según cree que mejor le va, yo me adecuo sin lugar a duda [...] (docente B, Ciencias Experimentales, UCM).

Por su parte, otros profesores destacan la versatilidad que debe caracterizar su trabajo en relación con las estrategias de aprendizaje de los estudiantes, pues a pesar de que planifican sigilosamente los objetivos, contenidos y actividades de enseñanza, también se debe considerar un grado de flexibilidad para ejecutar cambios en caso de situaciones imprevistas:

[...] Algunas veces tengo que cambiar sobre la marcha determinadas cosas, tienes que ir con tu clase planificada, pero también un poco cambiarla, adaptar alguna estrategia que no está funcionando, tener cierta versatilidad, capacidad de adaptación [...] (docente B, Lengua y Literatura, UCM).

Dentro de esta categoría, se identificaron 4 subcategorías de análisis:

a) Aprendizaje basado en el enfoque por competencias: algunos docentes recurren a los lineamientos conceptuales aportados desde el enfoque por competencias para explicar la promoción de estrategias de aprendizaje. En efecto, se percibe que el desarrollo de competencias en los alumnos debe guiarse según los conocimientos declarativos (asociado a la recepción pasiva de los contenidos y al aprendizaje individual de los estudiantes) y los 
procedimentales (más proclives a estrategias de aprendizaje grupales colaborativas y a un mayor protagonismo de los estudiantes) revisados en el marco de su materia:

[...] Trato de apuntar fundamentalmente al desarrollo de competencias y de tipos de aprendizajes: uno más de tipo conceptual declarativo, es el que trato de promocionar con la lección magistral... Y luego un conocimiento que tiene que ver más competencias aplicadas, lo que tradicionalmente en psicología lo conocemos por conocimiento procedimental, que es el que trato de implementar por la vía de actividades más aplicadas en grupos y demás [...] (docente C, Métodos de Investigación y Diagnóstico en Educación, UCM).

Otros docentes mencionan la importancia de considerar el fomento del autoaprendizaje y el desarrollo de competencias de aprendizaje autónomo, independiente y responsable entre los estudiantes:

[...] Un poco el autoaprendizaje o dijéramos, la responsabilidad del alumno, de que el alumno sea responsable de su propio aprendizaje en el sentido de bueno, de leer algo y de hacer algo y me gusta mucho trabajar en grupos, tengo en cuenta mucho el trabajo individual que se referiría un poco a las pequeñas tareas [...]" (docente C, Lengua y Literatura, UCM).

Por su parte, otros docentes mencionan el fomento de competencias socioafectivas, tales como el respeto, la tolerancia y el trabajo en equipo entre sus estudiantes: "[...] Respeto, también, si ahorita trabajan en grupo en clase y no logran fomentar el respeto y tolerancia, pues cuando estén afuera y trabajando ellos tienen que reflejar ese tipo de tolerancia [...]" (docente E, UABC).

b) Desarrollo del pensamiento crítico: otros docentes entrevistados aseveran que las estrategias de aprendizaje que intentan promover en sus estudiantes apuntan al desarrollo de la capacidad de análisis y argumentación crítica en torno a los contenidos educativos, destaca la trascendencia que algunos docentes otorgan al desarrollo de sus capacidades de expresión escrita y oral:

[...] La capacidad autónoma de pensamiento, esa es la capacidad de aprendizaje que quiero que saquen, es decir, que sepan analizar críticamente un texto, una película, un video, una canción, lo que sea, y sepan establecer un juicio propio, fundamentado, con 
argumentación. Entonces lo que busco es que sepan escribir con una estructura lógica interna, claro, con vocabulario preciso, que sepan argumentar su posición (docente B, Ciencias Sociales, UCM).

Además, según la percepción de algunos docentes se apunta a promover la capacidad de analizar y flexionar críticamente frente a la realidad social en general, y la realidad educativa en particular, en la que los estudiantes están insertos, de manera que sean capaces de generar cuestionamientos y juicios de valor al respecto:

[...]Que se enfrenten con una realidad en la que siempre han sido participantes pero que se enfrenten desde otro punto de vista, que empiecen a pensar en la escuela, pues que traten de enfrentarse a ese mundo críticamente, mi idea sería despertar el pensamiento crítico, sería la finalidad última, es despertar el pensamiento crítico para poder juzgar el hecho educativo de manera más sutil [...] (docentes A, Teoria e Historia, UCM).

c) Fomento del aprendizaje grupal colaborativo: la promoción de estrategias de aprendizaje relacionadas con el trabajo grupal entre estudiantes como vía de generación de significados y conocimientos compartidos fue una subcategoría de análisis recurrente dentro de la categoría de fomento de aprendizaje centrado en los estudiantes:

[...] Siempre lo he tenido muy claro y desde un principio llevo muchos años dando clase, he pensado que el aula tiene que ser una cooperación. Por lo tanto ese aprendizaje cooperativo, que a lo mejor en los últimos diez o quince años ha estado entre comillas muy de moda, yo llevo muchos años practicando... Primero les lleva al compromiso, a la disciplina de grupo, porque se comprometen con un grupo y si uno falla se reciente el grupo“ [...] (docente A, Ciencias Sociales, UCM).

En efecto, a partir de las percepciones de los docentes entrevistados, se mencionan la asignación y cumplimiento de roles y metas entre los miembros de los grupos de trabajo de estudiantes, así como la generación de debates, intercambio de ideas y atribuciones de sentido, a partir de la interacción grupal en torno a los contenidos educativos:

[...]Yo lo que hago principalmente es generar la participación en el estudiante... Es decir, que cualquier participación que él pueda tener, no evaluarla como buena o mala sino simplemente como es y conjuntamente con los demás compañeros o 
equipos, construir el aprendizaje, ya que el aprendizaje no se da en solitario sino en lo social $[\ldots]$ (docente C, UABC)

d) Aprendizaje situado: por último, algunos docentes apuntan a promover la construcción de conocimientos a partir de situaciones relacionadas con la cotidianidad y la experiencia diaria de los estudiantes. En este sentido, destacan que los propósitos principales en términos de promoción de estrategias de aprendizaje apuntan a buscar la reflexión y la construcción de significados desde la experiencia de los sujetos, refiriéndose de esta manera a los constructos asociados al desarrollo del aprendizaje situado:

[...]Hago muchísima labor emocional con ellos, poniendo actividades muy de la vida diaria, muy pragmáticas, que resultan vistosa, que resultan agradables, que reducen el tiempo de resolver una situación cuando te enfrentas a ella [...]" (docente A, Matemáticas, UCM); “[...] Procuro traerles experiencias cotidianas, me gusta mucho trabajar desde la experiencia que ellos tienen [...] (docente B, Matemáticas, UCM).

4.2 Aprendizaje centrado en los contenidos: otra categoría de análisis en torno a la promoción de estrategias de aprendizaje en los estudiantes se relaciona con la naturaleza del conocimiento enseñado; por lo tanto, su centro gira en torno a los contenidos educativos. Algunos docentes perciben que la orientación teórica o práctica de los contenidos asociados a sus materias influye en la promoción de estrategias de aprendizaje. De manera que el factor determinante sería la naturaleza de los contenidos. En efecto, algunos docentes aseveran que la revisión de contenidos teóricos implicaría un mayor fomento de estrategias individuales de aprendizaje, mientras que la realización de proyectos aplicativos implicaría una mayor promoción de los trabajos grupales colaborativos como estrategia de aprendizaje principal.

En este sentido, perciben que las estrategias de aprendizaje que promocionan en sus estudiantes varían de acuerdo con la materia que enseñan, orientándolas según la naturaleza conceptual (teórica) o práctica (metodológica) de los contenidos revisados:

[...] Varía por materia. Por ejemplo, la materia de Metodología, el 70\% de la materia ellos la imparten, la exponen, pero antes de que lleguen los muchachos a exponer frente al aula, frente a todos, antes ellos tienen que venir aquí conmigo a una asesoría, preparar su exposición, dictarme la exposición a mí, yo les hago preguntas específicas... En Procesos Grupales depende: cuando el contenido es muy teórico, 
les pido esquemas, cuando es práctico les pido que hagan casos [...] (docente $B$, $\mathrm{UABC})$.

4.3 Aprendizaje centrado en el docente: se debe destacar que una última categoría de análisis asociada a la promoción de estrategias de aprendizaje en los estudiantes remite al fomento del aprendizaje individual receptivo. En efecto, desde la percepción de algunos docentes entrevistados se puede observar una postura de corte más tradicional en cuanto a la enseñanza de los contenidos y las estrategias de aprendizaje que fomentan en sus estudiantes. Según su perspectiva, la incorporación de nuevos conocimientos debe seguir un camino receptivo a partir de los procesos de asimilación, memorización, ejercitación y análisis de la información (contenidos) que se le facilita al estudiantado. De esta manera, algunos docentes aseveran que la enseñanza de los contenidos educativos debe seguir una lógica de traspaso unidireccional del docente a los estudiantes: por un lado, el docente transmite la información y propone dinámicas de ejercitación y reforzamiento; por otro lado, los estudiantes deben aprender los contenidos a través de un proceso de incorporación individual, receptivo y pasivo:

[...] Recepción, asimilación, aprendizaje, memorización y análisis crítico de la documentación. No creo en el constructivismo. O sea, el constructivismo viene bien para determinar las áreas que están basadas en un golpe afectivo, una intuición, en una recreación artística. Aprender el concepto de relación educativa de lo que han dicho los clásicos, no puede basarse en una experiencia de construcción, eso viene en la asimilación final. ¿Qué dijo Aristóteles de la relación educativa? Lo que tienes que aprender es lo que dijo Aristóteles de la relación educativa y ahí no entra el constructivismo. Sí entra en la perspectiva particular que tú puedes tener sobre lo que dijo Aristóteles, pero yo diferencio en clase lo que es el aprendizaje del contenido y de lo que han dicho determinados autores, de lo que dice el alumno y lo que digo yo [...] (docente B, Teoria e Historia, UCM).

Seguidamente, en la Figura 1 se observa un esquema descriptivo de la dimensión estrategias de aprendizaje. Al respecto, se identificó una subdimensión relacionada con el fomento/promoción de estas estrategias en los estudiantes. Dentro de esta subdimensión, se levantaron 3 categorías de análisis (aprendizaje centrado en el estudiante, en los contenidos y en el docente) y 4 subcategorías de análisis (aprendizaje basado en el enfoque por 
competencias, desarrollo del pensamiento crítico, fomento del aprendizaje grupal colaborativo y aprendizaje situado), todas relacionadas con el fomento de estrategias de aprendizaje centradas en la actividad de aprendizaje de los estudiantes.

Figura 1.

Subdimensiones, categorías y subcategorías de análisis en torno a la dimensión estrategias de aprendizaje

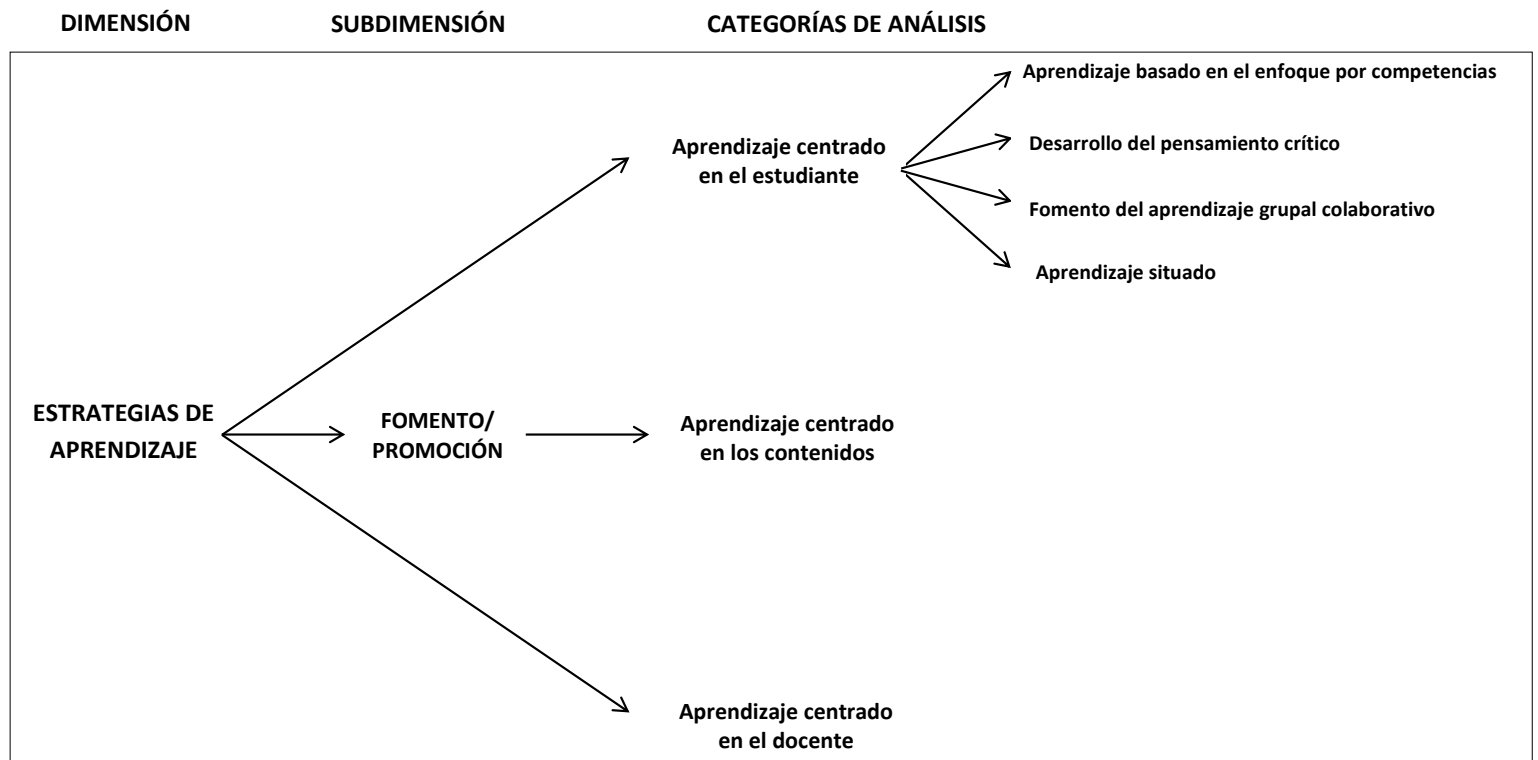

Fuente: elaboración propia (2017)

\section{Discusión}

La investigación en torno a las estrategias de aprendizaje ha producido una multiplicidad de delimitaciones conceptuales, de lo cual deriva una falta de precisión y de consenso evidentes en torno a este objeto de estudio (Díaz y Hernández, 2002; Donche et al., 2013; Fernández et al., 2011; García y Ortega, 2012; Kamboj y Kumar, 2015; León et al., 2014; Shukla y Dungsungnoen, 2016).

Dentro de estas propuestas conceptuales, la aportada por Díaz y Hernández (2002) ha sido de las más utilizadas y citadas en contextos de investigación educativa y de docencia en el nivel educativo superior. A partir de su comprensión como un conjunto de procedimientos que los estudiantes utilizan de modo intencional y reflexivo para la realización de las tareas escolares y el cumplimiento de los objetivos trazados por los docentes, los autores plantean un amplio abanico de técnicas (repetición simple y acumulada, uso de palabras claves, imágenes, resúmenes o analogías, identificación de categorías, redes semánticas, mapas 
conceptuales) a partir de los procesos cognitivos implicados en dichos procedimientos y las finalidades que persiguen los estudiantes a través de su ejecución.

Frente a lo anterior, el presente documento intenta aportar una nueva perspectiva teórica para comprender las estrategias de aprendizaje en contextos educativos a través de dos componentes principales: i) el centro de interés en el que gira el desarrollo del aprendizaje de los estudiantes y ii) la relación íntima e inseparable entre las estrategias de aprendizaje y la acción de enseñanza (estrategias) de los docentes. De esta manera, aporta una forma novedosa de caracterizar las estrategias de aprendizaje, más allá del conjunto de técnicas que tradicionalmente se han puntualizado para comprenderlas.

En efecto, en concordancia con la propuesta de Coll (2004), se arguye que las prácticas educativas formales se pueden caracterizar a partir de un triángulo interactivo o didáctico cuyos vértices son: i) contenidos educativos (objeto de la enseñanza y el aprendizaje), ii) actividad de enseñanza del docente (apoyo sistemático y ajustado al proceso de aprendizaje por parte de la acción docente) y iii) actividad de aprendizaje de los estudiantes (atribución de sentido y construcción de significados en torno a los contenidos educativos). Justamente, las estrategias relacionadas con este último vértice se ven permeadas por el punto de interés que elija el agente de enseñanza (docente) para promoverlas y fomentarlas.

A partir de la información recolectada en las entrevistas, se puede observar que las estrategias de aprendizaje que los docentes intentan fomentar en sus alumnos se delinean según el foco de interés que se ponga en algunos de los 3 vértices destacados por Coll (2004). Mientras algunos docentes centran su interés en la actividad constructiva de los estudiantes y a partir de ahí promueven estrategias relacionadas con el protagonismo de ellos (proyectos de investigación, trabajo colaborativo, participación activa en clases), otros docentes ponen su atención en su actividad de enseñanza, la transmisión de contenidos educativos y el fomento de estrategias de aprendizaje de retención, repetición y memorización de los cuerpos de información asociados a sus materias. Finalmente, otros docentes centran su interés en el tipo de contenido enseñado y a partir de ahí promueven estrategias de aprendizaje basadas en un rol más activo y protagónico de sus estudiantes (conocimientos prácticos o procedimentales) o en un rol más pasivo y receptivo (conocimientos teóricos o declarativos).

Múltiples estudios (Abdi, Lael y Ahmadyan, 2013; Donche et al., 2007; Fernández et al., 2011; García et al., 2012; Gaston et al., 2016; Kablan y Kaya, 2014; Monereo et al., 2007; Shukla y Dungsungnoen, 2016) han destacado la trascendencia de los docentes para 
fomentar estrategias de aprendizaje activas y efectivas en sus estudiantes, así como la influencia y el impacto de las estrategias de enseñanza en su proceso de aprendizaje. En el presente artículo se argumenta que la enseñanza y el aprendizaje conforman un binomio inseparable en torno a los contenidos educativos, de manera que no se puede, ni se debe, hablar de estrategias de enseñanza y estrategias de aprendizaje como dos fenómenos independientes y separados. La acción de enseñanza del docente tiene su razón de existir en el proceso de aprendizaje de los estudiantes: no se puede comprender un proceso de aprendizaje sin el acompañamiento sistemático y planificado por parte de los docentes. Asimismo, el aprendizaje escolarizado de alcances significativos surge como consecuencia de la tutoría, facilitación y monitoreo de un profesor, de manera que si bien no se descartan procesos de autoaprendizaje (o aprendizaje autodidáctico), en el contexto de la educación escolarizada la influencia del actuar docente es innegable.

De esta manera, desde la percepción de los docentes entrevistados se vislumbra una significación del aprendizaje como un proceso que puede estar determinado por tres elementos principales: i) por su acción de enseñanza, de lo cual deriva una categoría de aprendizaje receptivo y pasivo, con objetivos eminentemente memorísticos, retentivos y/o repetitivitos; ii) por el tipo de conocimiento involucrado, distinguiéndose principalmente entre conocimientos teóricos (aprendizaje individual receptivo) y conocimientos prácticos (aprendizaje grupal participativo); y iii) por las características cognitivas de los estudiantes, de lo cual deriva una comprensión del aprendizaje como un proceso versátil y flexible según la multiplicidad de estilos cognitivos y la eventualidad de aspectos no considerados en su planificación de enseñanza.

Esta última interpretación del aprendizaje se identifica con los postulados aportados desde las corrientes constructivistas que intentan explicar los procesos de generación de conocimientos en los individuos en contextos educativos. En efecto, ciertos elementos constitutivos del enfoque por competencias (actualmente en boga dentro del ámbito de la educación), el desarrollo del pensamiento crítico y autónomo frente a la realidad social en general (y educativa en particular), el fomento del aprendizaje grupal colaborativo entre los estudiantes para construir significaciones socio-culturales comunes, así como la promoción de procesos de aprendizaje situados en el contexto real y cotidiano en el que se desenvuelven los estudiantes, son algunas de las propuestas que se han venido erigiendo para contrarrestar la presencia de enfoques educativos tradicionales, identificados principalmente con interpretaciones conductistas en torno a la enseñanza y el aprendizaje 
(Díaz y Hernández, 2002; Gardner, 2008; Rosas y Balmaceda, 2008; Serrano GonzálezTejero y Pons Parra, 2011; Tünnermann Bernheim, 2011).

Se espera que estas perspectivas de análisis sirvan de base para profundizar la comprensión de las estrategias de aprendizaje fomentadas en contextos de formación de futuros agentes educativos, principalmente, aquellas relacionadas con la acción constructiva de conocimientos por parte de los estudiantes con base en sus características cognitivas y metacognitivas. Finalmente, hay que destacar que el presente documento reporta los principales hallazgos de una investigación que no se propuso obtener conclusiones generalizables a partir de su muestra de estudio. En efecto, se debe mencionar que una limitante innegable fue la selección de una muestra poblacional intencional no representativa, por lo cual no se podrían generalizar sus resultados ni establecer conclusiones tajantes y absolutas. Queda el desafío por delante de contrastar estos hallazgos con los aportes de otros estudios que utilicen muestras representativas de población y ofrezcan análisis e indicadores cuantitativos más objetivos, los cuales se puedan triangular con las categorías de análisis destacadas con base en las percepciones de los docentes entrevistados en esta investigación.

\section{Referencias}

Abdi, Alí, Lael, Soosan y Ahmadyan Hamze. (2013). The Effect of Teaching Strategy Based on Multiple Intelligences on Students' Academic Achievement in Science Course. Universal Journal of Educational Research, 1(4), 281-284. Recuperado de http://files.eric.ed.gov/fulltext/EJ1053949.pdf

Carbajosa, Diana. (2011). Debates desde paradigmas en la evaluación educativa. Revista Perfiles Educativos, 33(128), 183-192.

Coll, César. (2004). Psicología de la educación y prácticas educativas mediadas por las tecnologías de la información y comunicación. Una mirada constructivista. Revista Sinéctica, (25), 1-24. Recuperado de http://www.redalyc.org/articulo.oa?id=99815899016

Díaz, Frida y Hernández, Gerardo. (2002). Constructivismo y aprendizaje significativo. En Estrategias docentes para un aprendizaje significativo (pp. 23-62). México: McGrawHill.

Donche, Vicent, De Maeyer, Sven y Van Petegem, Peter. (2007). Teachers Conceptions of Learning and Teaching and Their Effect on Student Learning. British Educational Research Association Anual Conference, Institute of Education, University of London, 5-8 September. Recuperado de http://www.leeds.ac.uk/educol/documents/166752 
Donche, Vicent, De Maeyer, Sven, Coertjens, Liesje, Van Daal, Tine y Van Petegem, Peter. (2013). Differential use of learning strategies in first-year higher education: The impact of personality, academic motivation, and teaching strategies. British Journal of Educational Psychology, 83, 238-251. Recuperado de https://www.ncbi.nlm.nih.gov/pubmed/23692533

Fernández Nistal, María Teresa, Pérez Ibarra, Ricardo Ernesto, Peña Boone, Sergio Humberto y Mercado Ibarra, Santa Magdalena. (2011). Concepciones sobre la enseñanza del profesorado y sus actuaciones en clases de ciencias naturales de educación secundaria. Revista Mexicana de Investigación Educativa, 16(49), 571-596. Recuperado http://www.redalyc.org/pdf/140/Resumenes/Resumen 14018533011 1.pdf

García Salas, Lilia y Ortega Massé, Juan Carlos. (2012). Influencia de los estilos de aprendizaje y la autorregulación en el rendimiento escolar de los alumnos de Bachillerato a Distancia del Estado de México. Revista Mexicana de Bachillerato a Distancia, 4(7), 122-131. Recuperado de http://www.revistas.unam.mx/index.php/rmbd/article/view/45262

García Cué, José Luis, Sánchez Quintanar, Concepción, Jiménez Velázquez, Mercedes Aurelia y Gutiérrez Tapias, Mariano. (2012). Estilos de aprendizaje y estrategias de aprendizaje: un estudio en discentes de postgrado. Revista Estilos de Aprendizaje, 10(10), 1-17. Recuperado de http://www2.uned.es/revistaestilosdeaprendizaje/numero 10/articulos/Articulo06.pdf

Gardner, Howard. (2008). La educación de la mente y el conocimiento de las disciplinas. Barcelona: Paidos.

Gastón, Alishia, Martínez, James y Martín, Ellice. (2016). Embedding Literacy Strategies in Social Studies for Eighth-Grade Students. Journal of Social Studies Education Research, 7(1), 73-95. Recuperado de http://dergipark.gov.tr/download/articlefile/264153

Hernández Rojas, Gerardo. (1998). Paradigmas en psicología de la educación. México D.F.: Paidós.

Kablan, Zeynel y Kaya, Sibel. (2014). Preservice Teacher's Constructivist Teaching Scores Based on Their Learning Styles. Australian Journal of Teacher Education, 39(12), 6677. Recuperado de http://ro.ecu.edu.au/cgi/viewcontent.cgi?article=2459\&context=ajte

Kamboj, Pooja y Kumar Singh, Sushil. (2015). Effectiveness of Selected Teaching Strategies in Relation to the Learning Styles of Secondary School Students in India. Interchange, 46(3), 289-312. Recuperado de https://link.springer.com/content/pdf/10.1007\%2Fs10780-015-9253-7.pdf

León Urquijo, Ana Patricia, Risco del Valle, Eduardo y Alarcón Salvo, Cristina. (2014). Estrategias de aprendizaje en educación superior en un modelo curricular por competencias. Revista de Educación Superior 43(172), 123-144. Recuperado de http://publicaciones.anuies.mx/pdfs/revista/Revista172 S3A6ES.pdf 
Monereo, Carles, Castelló, Monserrat, Clariana, Marcè, Palma, Monserrat y Pérez, María. (2007). Estrategias de enseñanza y aprendizaje. Formación del profesorado y aplicación en la escuela. México D.F.: Graó:

Piñuel Raigada, José Luis. (2002). Epistemología, metodología y técnicas de análisis de contenido. Estudios de Sociolingüística 3(1), 1-42. Recuperado de https://www.ucm.es/data/cont/docs/268-2013-07-29-

Pinuel Raigada AnalisisContenido 2002 EstudiosSociolinguisticaUVigo.pdf

Robles, Bernardo. (2011). La entrevista en profundidad: una técnica útil dentro del campo antropofísico. Cuicuilco, 18(52), 39-49. Recuperado de http://www.redalyc.org/pdf/351/35124304004.pdf

Rodríguez Garza, Mayela del Refugio, Sanmiguel Salazar, Francisca y Hernández Vallejo, Claudia. (2012). Los estilos de aprendizaje y la aplicación de estrategias de enseñanza-aprendizaje para favorecer el rendimiento académico en un posgrado de educación mexicano. En Fernando Guerra, Rosa García-Ruiz, Natalia González, Paula Renés y Ana Castro (Coords.), Estilos de aprendizaje: investigaciones y experiencias (pp. 1-9). Congreso Mundial de Estilos de Aprendizaje, Santander, España. Recuperado de https://dialnet.unirioja.es/servlet/articulo?codigo=4664795

Rosas, Ricardo y Balmaceda, Christian. (2008). Piaget, Vigotsky y Maturana: constructivismo a tres voces. Buenos Aires: Aique.

Serrano González-Tejero, José Manuel y Pons Parra, Rosa María. (2011). El constructivismo hoy: enfoques constructivistas en educación. Revista Electrónica de Investigación Educativa 13(1). Recuperado de http://redie.uabc.mx/index.php/redie/article/viewFile/268/431

Shukla, Divya y Dungsungnoen, Aj Pattaradanai. (2016). Student's Perceived Level and Teacher's Teaching Strategies of Higher Order Thinking Skills; A Study on Higher Educational Institutions in Thailand. Journal of Education and Practice, 7(12), 211-219. Recuperado de https://eric.ed.gov/?id=EJ1099486

Tünnermann Bernheim, Carlos. (2011). El constructivismo y el aprendizaje de los estudiantes. Universidades, (48), 21-32. Recuperado de http://www.redalyc.org/articulo.oa?id=37319199005

Vargas Jiménez, Ileana. (2012). La entrevista en la investogación cualitativa. Nuevas tendencias y retos. Revista Caliad en la Educación Superior, 3(1), 119-139. Recuperado

de http://biblioteca.icap.ac.cr/BLIVI/COLECCION UNPAN/BOL DICIEMBRE 2013 69/UN ED/2012/investigacion cualitativa.pdf 